\title{
Considerations for using ZigBee technology in vehicular non-critical applications
}

\author{
Valentin Iordache, Marius Minea, Razvan Andrei Gheorghiu \\ Transport Faculty, POLITEHNICA University of Bucharest \\ Bucharest, Romania \\ Email: \{valentin.iordache, marius.minea, andrei.gheorghiu\}@upb.ro
}

\begin{abstract}
The paper presents results of ZigBee communication tests performed in a specifically set electromagnetic environment, with the purpose to determine the applicability of ZigBee technology in non-critical messaging for vehicular communications. Known for its low energy consumption, the ZigBee technology might be used in background messaging for cooperative driving, with the purpose to reduce the overload on the main channels used for emergency message warning, or other critical applications. In the paper are presented the test bed, results and solutions for new approaches with usability to vehicular communications.
\end{abstract}

\section{INTRODUCTION}

$\mathrm{L}$ ATELY, the recent development of vehicular communications towards information exchange between moving vehicles and road infrastructure lead to a significant increase of interferences, especially in the 2.4 $\mathrm{GHz}$ band, where channels are shared with numerous access points and other devices outside road traffic domain. Except DSRC (Dedicated Short-Range Communications), which employs other frequencies, in several applications, such as traffic sensor wireless networks, vehicle counting/identification and vehicular communications, the use of Wi-Fi or ZigBee technologies has triggered a lot of solutions, partly tested, partly still under development. Therefore, the coexistence of many $2.4 \mathrm{GHz}$ devices operating in close vicinity has become very challenging and numerous studies have been carried on in this direction.

The layers MAC (media access control) and PHY (physical) late specifications for low-rate wireless personal area networks (PAN), IEEE 802.15.4 using 2.4 GHz for the ISM (Industrial, Scientific and Medical) band has been developing in a high rhythm in the recent years. The ZigBee communications technology, based on IEEE 802.15.4, has also been deeply investigated. There can be seen an increasing demand of communications on short distance. Related to that two important industrial wireless network standards based on IEEE 802.15.4, Wireless HART, and

This work has been funded by University Politehnica of Bucharest, through the "Excellence Research Grants" Program, UPB - GEX. Identifier: UPB-EXCELENTT̆-2016 Research project title "VEHINET - Rețea cu conținut informațional adaptiv la condițiile mediului destinată deplasării inteligente a vehiculelor", Contract number 45/26.09.2016 (acronym: VEHINET).
ISA100 have been approved. These wireless networks use the same $2.4 \mathrm{GHz}$ ISM band; moreover, as a license-free radio band, $2.4 \mathrm{GHz}$ ISM has also been widely employed by many non-IEEE 802.15.4 wireless networks, so coexistence among them must be also considered when developing new applications that share the same frequencies. The following section briefly presents the most recent advances in this area, based on a literature study.

\section{II.ZIGBEE - WI-FI COEXISTENCE - LITERATURE SURVEY}

When several devices try to communicate in the same bandwidth, in different or overlapping channels, there are some important questions that a researcher should ask:

- which parameter is more adequate for investigating the electromagnetic compatibility?

- is the interference phenomenon experienced with the same intensity for both communicating devices?

- is the sense of communication (uplink / downlink) affected similarly in case of a disturbance?

- in which way position of devices, direct line of sight, reflections and refractions, antennas' position etc. do affect the coverage and quality of communication?

- is it possible to create adequate models applicable in case of studying communication quality for two or more devices?

The worst case is when communication is severely affected by interference and there is a lot of message packets loss, message delaying, and bandwidth consumption. As showed by the authors in [1], packet error rate is more than $90 \%$ when severe interference occurs. An answer to the last question put above is partly given by the authors of [2], who present the effect of different orientations of IEEE $802.11 \mathrm{n}$ transmission on IEEE 802.15.4 devices.

Regarding the elements that should be studied in a complete interference test, the authors of [3] gave a comprehensive solution for the input parameter, the output parameter and for the behavior sets.

As also observed by the authors of [4], the bandwidth of the IEEE $802.11 \mathrm{~b}$ is $22 \mathrm{MHz}$, eleven times larger than the one of IEEE 802.15.4, which is $2 \mathrm{MHz}$. When Wi-Fi and ZigBee transmission coexist, usually every collision between a Wi-Fi packet and a ZigBee packet results in the ZigBee packet being lost. 
Beneficiating of a bandwidth of only $2 \mathrm{MHz}$, ZigBee CCA captures the full power of other ZigBee transmissions in the same channel, but only $2 / 22$ th $-($ or $-10.4 \mathrm{~dB}$ ) - of the Wi-Fi transmitted power, resulting in a $9.6 \mathrm{~dB}$ higher sensitivity to Wi-Fi than to ZigBee.

Most of scientific papers' authors conclude after different experiments that ZigBee is oversensitive to $\mathrm{Wi}-\mathrm{Fi}$, while Wi-Fi is insensitive to ZigBee beyond a Heterogeneous Exclusive Clear Channel Assessment (CCA) Range, which is calculated by Zhen et al. in [5] to be $25 \mathrm{~m}$ with the free space path loss.

Tytgat et al. in [6] demonstrate that the deployment of a $\mathrm{CACCA}^{1}$ protocol achieves substantial reduction of the ZigBee incurred packet loss, without needing any additional information exchange (and the incurred overhead), nor having a severe impact on the energy consumption. CACCA concept enables Wi-Fi to detect ZigBee presence and to reduce channel interference in different implementations.

The ZigBee technology is mainly used for low data-rate applications such as home automation, or smart-grid metering and demand response. The ZigBee Alliance defines an interference mitigation technique, named Frequency Agility mechanism [7], that can be divided into three phases: interference detection, channel evaluation and interference mitigation [8].

The authors conclude that the ZigBee Frequency Agility interference detection threshold is a crucial parameter that needs to be carefully set. In most cases, ZigBee Frequency Agility mechanism successfully switches the ZigBee network to a channel with the lowest interference level, but it can only successfully mitigate the interference that occupies a fraction of the ISM $2.4-\mathrm{GHz}$ band and may be inadequate for the interference that emits signals throughout the entire band.

\section{ZIGBEE MESSAGE TRANSFER TESTS - PROCEDURE OVERVIEW}

To determine the usability of low-energy ZigBee technology for vehicular communications (e.g., cooperative driving), a test setup has been deployed. The purpose of the tests is to assess the capabilities of the ZigBee link to maintain and transfer enough bandwidth to allow for a normal (non-emergency) messaging between moving vehicles and road infrastructure, in a typical Wi-Fi urban environment. The reason to employ such a setup is that on an external motorway - outside urban areas - the probability to encounter interfering $\mathrm{Wi}-\mathrm{Fi}$ APs is much lower than in the cities.

Message transfer time measurements were initially performed inside the University building, in open space, with clear line of sight between the communicating modules. Interferences were created using a $\mathrm{Wi}-\mathrm{Fi}$ router and a computer connected to it, both in the proximity of the
ZigBee modules, by transferring large files with speeds between $40 \mathrm{Mbps}$ and $70 \mathrm{Mbps}$. A common environment with unknown interferences was chosen instead of an interference free one, to resemble with similar situations in a real urban vehicular environment.

If we consider the channel distribution, ZigBee channel 26 is furthest from any Wi-Fi channel overlapping, and less likely to be influenced by any Wi-Fi traffic, so it was used for message transfer time measurement.

The following scenarios were taken into consideration:

- The presence of a typical background environmental noise, produced by Wi-Fi APs with connected devices in a University;

- Heavy traffic on Wi-Fi channel 1;

- Heavy traffic on Wi-Fi channel 6;

- Heavy traffic on Wi-Fi channel 11;

- Heavy traffic on Wi-Fi channel 13;

- Heavy traffic on ZigBee channel 26.

The reason the authors decided to set up the router to use channels 1,6 and 11 is that they are the only nonoverlapping channels, and many Wi-Fi networks are using them by choice as mentioned in [9].

Wi-Fi channel 13 was chosen because is the closest one to ZigBee channel 26, and interferences because of it are most likely to appear.

For the last scenario considered, another pair of ZigBee modules was employed, set on the same communication channel as the ones used for measurements, and transmitting data with a high rate, similarly to the case where other vehicles might use the same channel.

Four XBee S2 modules were used. The criteria for selecting these modules were their affordable price and the high availability. Each module was connected to an Arduino Uno board with an XBee Shield.

Each of the two XBee pairs had one module set as a Coordinator and the other as an End-Device. One pair was employed for message transfer time measurements and the other one to generate interferences on channels 26 .

The router and a computer, as well as the second pair of XBee modules were positioned in the middle, between main XBee modules, to be able to maintain constant speed when creating interference. The RF environment was considered to be similar to an average urban location, with Wi-Fi Access Points from different locations like offices, residential buildings, road infrastructure equipment or mobile devices. The distance between main XBee modules was modified between 0 and 50 meters, with a 5-meter step. A longer distance of 55 meters was also tested, but there was little to no connection between modules, even in presence of typical background environmental noise.

Results of the performed tests are presented in the following section.

\footnotetext{
${ }^{1}$ CACCA - Coexistence Aware Clear Channel Assessment
} 


\section{ZiGBEE MESSAGES TRANSFER TIME MEASUREMENTS}

After connecting, the Coordinator started to transmit data to the End-Device, which in return responded with the same amount of data.

The message transfer time was then measured between a clear send and a correct received response, for 100 consecutive tests. Three different cases have been approached, using messages with 256, 512 and 1024 bits per segment.

As it can be seen from Fig. 1, Fig. 2 and Fig. 3 traffic on channels 1, 6 and 11 had no influence over the message transfer time, compared with the values measured in presence of typical background environmental noise, for each of the three considered cases. This corresponds to the expectations because Wi-Fi channels 1, 6 and 11 do not overlap with ZigBee channel 26. Also, because bigger messages will require more time for them to be sent, no matter if there are interferences or not, the message transfer time increased as the size of the message increased, with average values being around 59, 149 and 188 milliseconds corresponding to a message length of 256, 512 and 1024 bits. Due to lack of interferences between these channels, the authors stopped testing these scenarios for distances greater than 25 meters, considering that values obtained in the presence of typical background environmental noise would be sufficient to describe them all.

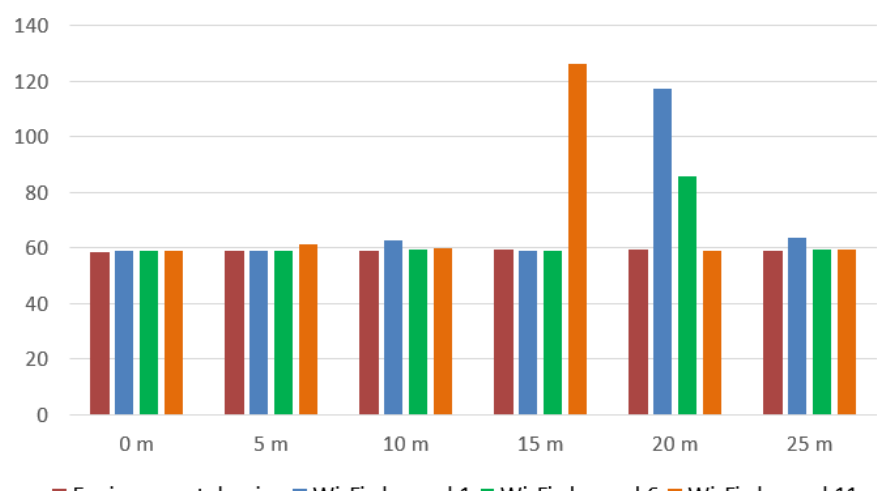

Environmental noise $\square$ Wi-Fi channel 1 Wi-Fi channel 6 wi-Fi channel 11

Fig. 1. Average message transfer time (ms) for 256 bit message with traffic on specified Wi-Fi channels

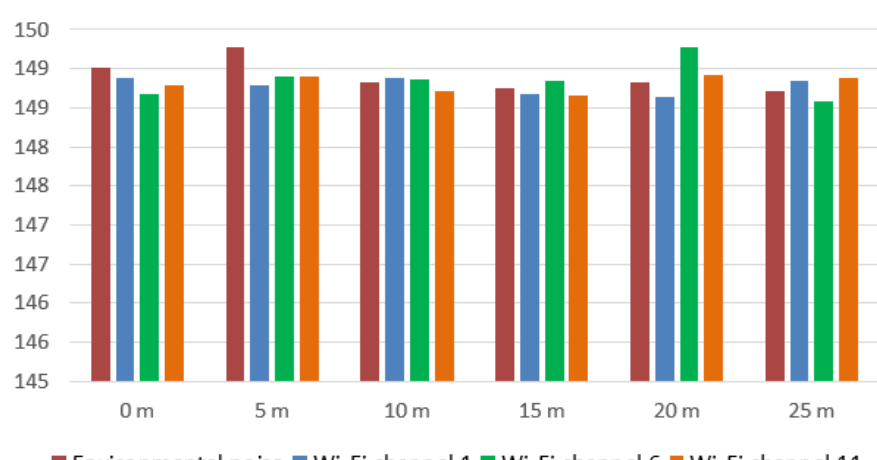

Fig. 2. Average message transfer time (ms) for 512 bit message with traffic on specified Wi-Fi channels

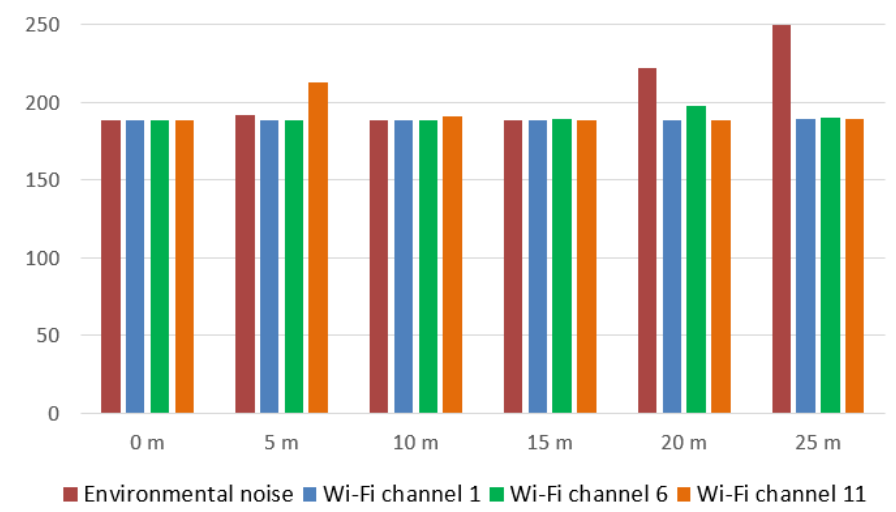

Fig. 3. Average message transfer time (ms) for 1024 bit message with traffic on specified Wi-Fi channels

In Fig. 4, Fig. 5 and Fig. 6 it can be seen that traffic on channel 13 had the highest influence over the message transfer time for each of the three cases with an average raise of about 610, 370, and respectively 154 percent (compared with the value measured in presence of typical background environmental noise). Under the same conditions, traffic created by another ZigBee pair of devices on the same channel 26 had less influence, message transfer times having average raises of about 60, 12 and respectively 11 percent.

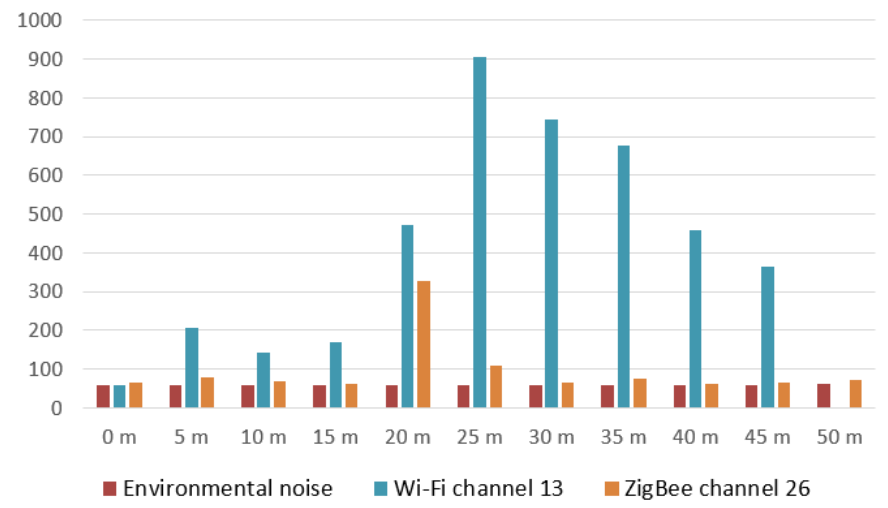

Fig. 4. Average message transfer time (ms) for 256 bit message with traffic on specified channels

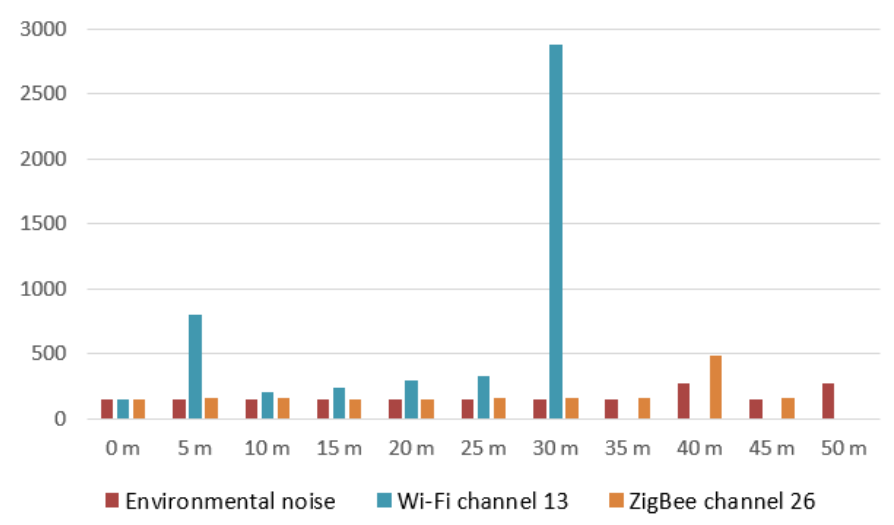

Fig. 5. Average message transfer time (ms) for 512 bit message with traffic on specified channels 


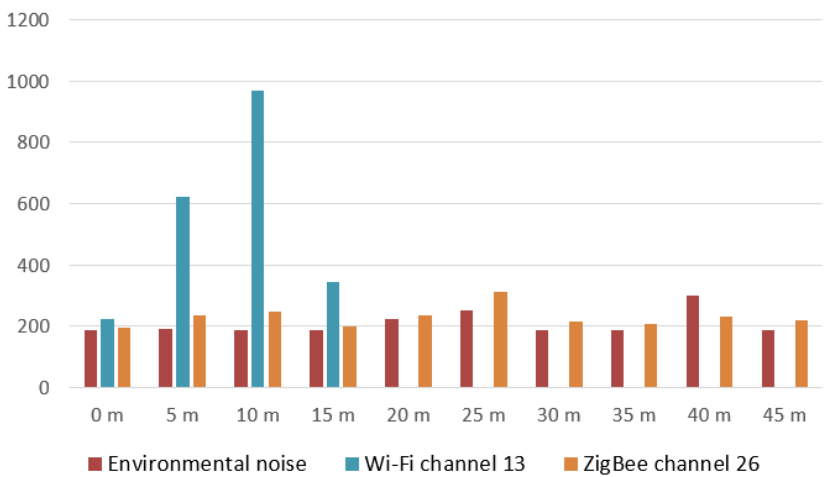

Fig. 6. Average message transfer time (ms) for 1024 bit message with traffic on specified channels

Increasing distance between devices will lead, obviously, to the situation in which communication would be impossible. As expected, it will happen first for greater message lengths.

For 1024 bit messages under typical noise conditions or with another ZigBee on channel 26 the maximum distance is 45 meters, and drops considerably to 20 meters if traffic on Wi-Fi channel 13 is present.

When using messages with 512 bits of data, maximum communication distance does not improve significantly: 50 meters with environmental noise, 45 meters with another ZigBee on channel 26, and 30 meters with traffic on Wi-Fi channel 13.

Smaller messages are transferred with more success in a perturbated environment, as for 256 bit messages the maximum distance is increased to 45 meters with traffic on Wi-Fi channel 13, and to 50 meters in the other two cases.

Regarding maximum values, for a data transfer affected by a typical environmental noise or traffic on Wi-Fi channels 1,6 or 11 , it resulted that they have reasonable values: around 65 milliseconds for 256 bit messages, 153 milliseconds for 512 bit messages and 196 milliseconds for 1024 bit messages.

For a data transfer affected by traffic on Wi-Fi channel 13, no matter the distance or length of message, maximum values for the transfer time proved to be too high to be suitable for critical applications in a vehicular environment (values between 1 and 7 seconds, mostly around 3 or 4 seconds).

In the last case, a data transfer affected by traffic on the same ZigBee channel, maximum values were lower than in the previous case, around 250 milliseconds for 256 bit messages, 320 milliseconds for 512 bit messages and 570 milliseconds for 1024 bit messages, but they may also be considered too high for some applications or vehicle speeds.

\section{V.CONCLUSION}

ZigBee communication is heavily influenced by Wi-Fi in the proximity. As the test program measured the transfer time for a two-way non-erroneous communication, the XBee modules were unable to obtain any result as the distance between them increased, when using data transfer on Wi-Fi channel 13, which use the closest frequency band to ZigBee channel 26.

To conclude, for distances up to 50 meters, exchange of messages between vehicle and infrastructure will be achieved in a fairly good amount of time, at reasonable travel speeds or for short time stationary vehicle, if we consider a low handshake time between ZigBee modules (which is typical for this technology) and low interferences from Wi-Fi traffic on channel 13 (situation that has a low probability of occurrence because channels 1,6 or 11 are usually preferred) and ZigBee traffic on channel 26 (that can be avoided in non-crowded areas). As a result, implementation of ZigBee communications in a vehicular environment would be possible if one previously determines the criticalness of the desired applications and considers measuring and determination of the level of interference present in the areas where applications are to be implemented.

\section{REFERENCES}

[1] A.Sikora, V. F. Groza. Coexistence of IEEE802.15.4 with other systems in the $2.4 \mathrm{GHz}-\mathrm{ISM}$-band. Proceedings of the IEEE Instrumentation and Measurement Technology Conference (IMTC '05) May 2005, DOI: 10.1109/IMTC.2005.1604479;

[2] S. Y. Shin, S. Choi, H. S. Park, W. H. Kwon. Lecture notes in computer science: packet error rate analysis of IEEE 802.15.4 under IEEE $802.11 \mathrm{~b}$ interference. Proceedings of the 3rd International Conference on Wired/Wireless Internet Communications (WWIC '05) May 2005, DOI: 10.1007/11424505_27;

[3] D. Yang, Y. Xu, M. Gidlund. Wireless Coexistence between IEEE 802.11- and IEEE 802.15.4-Based Networks: A Survey. International Journal of Distributed Sensor Networks. July 2011, DOI:10.1155/2011/912152;

[4] R. E. Ziemer, R. L. Peterson, D. E. Borth. Introduction to Spread Spectrum Communications. 1995 New York, NY, USA Prentice Hall Google Scholar;

[5] B. Zhen, H-B. Li, S. Hara, R. Kohno. Clear channel assessment in integrated medical environments. EURASIP J. Wirel. Commun. Netw. vol. 2008. (2008), DOI:10.1155/2008/821756

[6] L. Tytgat, O. Yaron, S. Pollin, I. Moermann, P. Demeester. Avoiding collisions between IEEE 802.11 and IEEE 802.15.4 through coexistence aware clear channel assessment. Journal on Wireless Communications and Networking, December 2012, DOI: 10.1186/1687-1499-2012-137;

[7] ZigBee Alliance, ZigBee Specification Document 053474r17, 2008;

[8] Adib Sarijari Mohd, Sharil Abdullah Mohd, Anthony Lo, Rozeha A. Rashid. Experimental Studies of the ZigBee Frequency Agility Mechanism in Home Area Networks. IEEE 39th Conference on Local Computer Networks Workshops (LCN Workshops), 8-11 Sept. 2014, Edmonton, AB, Canada, DOI: 10.1109/LCNW.2014.6927725.

[9] R.A. Gheorghiu, V. Iordache, Analysis of vehicle to infrastructure (V2I) communication efficiency using the ZigBee protocol. Proceedings of the third International Conference on Traffic and Transport Engineering, November 24-25, Belgrade, Croatia, 2016 